\title{
Influence of dielectric constant of polymerization medium on processability and ammonia gas sensing properties of polyaniline
}

\author{
PARTHA PRATIM SENGUPTA, PRADIP KAR and BASUDAM ADHIKARI* \\ Materials Science Centre, Indian Institute of Technology, Kharagpur 721 302, India
}

MS received 9 January 2008; revised 11 January 2011

\begin{abstract}
Polyaniline (PANI) was synthesized by the oxidation of aniline hydrochloride in the presence of ammonium persulphate and hydrochloric acid. The polymerization reaction was carried out in several batches in different solvent media by changing the volume ratio of $N, N$-dimethyl formamide (DMF) and water as binary solvent mixture. The dielectric constant of the polymerization medium for each batch reaction was determined by measuring the capacitance with change in frequency. The UV spectra of the synthesized polyaniline solutions helped us to optimize the ratio of the binary solvent to get sufficient polymer growth and processability. Thin film of processable polyaniline was then deposited on glass slides coated with polyvinyl alcohol (PVA) crosslinked with maleic anhydride (MA). FTIR and XRD studies of the coated film were also done. AFM studies further helped in the morphological study of the film deposited. Finally, conductivity and ammonia gas-sensing property of the polyaniline film were also studied.
\end{abstract}

Keywords. Dielectric constant; processability; conductivity; gas sensing.

\section{Introduction}

The dielectric constant is a macroscopic property, which plays a significant role in the solution properties (Smith 1955; Dannhauser and Flucckinger 1964; Janini and Katrib 1983) of the reaction medium. It is a useful technique in characterizing molecular ordering in solutions and the value of the dielectric constant is strongly related to the chemical structure of a molecule and to its intermolecular interactions (Smyth 1980; Ghanadzadeh and Beevers 2002, 2003; Ghanadzadeh et al 2003). For this reason, this technique has been used extensively in condensed matter physics (Janini and Katrib 1983), polymer science (Dannhauser and Flucckinger 1964) and materials science (Smith 1955; Dannhauser and Flucckinger 1964; Janini and Katrib 1983) etc. The measurement of a.c. conductivity and dielectric constant of $\mathrm{HCl}$-doped PANI in the temperature range of 77$410 \mathrm{~K}$ and in the frequency range of $100 \mathrm{~Hz}$ to $1 \mathrm{MHz}$ was done by Singh et al (1999). The dielectric constant was found to be crucially dependant on the $\mathrm{pH}$, doping level, frequency and temperature. Hence the dielectric constant of the synthesized conducting polymers can be an important property in developing a processable polymer. We know that the fabrication of good quality films of conducting polymer is often hampered by solubility problems. Long-term mechanical and chemical stability of the polymeric materials are points of concern to make a successful sensor. A useful approach for the improvement of the processability of conducting polymers involves blending with suitable matrix

*Author for correspondence (ba@ matsc.iitkgp.ernet.in) polymers (Jousseaume et al 1998a, b; Planes et al 1998). An ammonia sensor based on conducting polypyrrole (PPy) was one of the early practical realizations of conducting polymer sensors. Its sensitivity, however, was relatively low and the response was not very reversible (Nylander et al 1983).

PVA possesses some advantages including its flexible molecular chains, high strength, good adhesion to electrodes and its ductile nature. Ojio and Miyata (1986) had prepared PPy-PVA films by electrochemical polymerization. PPy-PVA films prepared by electrochemical polymerization onto a precoated PVA matrix were studied by Lindsey and Street (1984). These studies concluded that PPy-PVA composite film combines the advantageous mechanical properties of the host polymer with the electrical properties of PPy. However, such synthesis of electroactive polymer onto an insulating host matrix by electrochemical polymerization has other disadvantages.

Polyaniline was found to be a better choice for gases such as ammonia because of its higher sensitivity, reversible response and shorter response time. Ammonia sensors based on doped polyaniline films have been extensively studied (Kukla et al 1996; Dhawan et al 1997; Koul et al 2001; Chabukswar et al 2001; Deng et al 2002; Huang L M et al 2003). In spite of the various advantages of polyaniline as ammonia gas sensor, some fundamental problems persist. Sensitivity of the responses is not always satisfactory. However, there is a considerable scope of improvement of reversibility and reproducibility of the processes involved in the sensing action. The emeraldine salt obtained after synthesis is stable in air, but the loss of its conductivity happens with moisture 
or heat. Moisture is a perennial problem affecting the electrical property of conducting polymer (Tobolkova et al 2004) and with heat it is believed that the dopant species $(\mathrm{HCl})$ are so small that they can be evaporated or sublimated out of the polymer (Sun et al 1994; Chen and Lee 1995). In order to overcome the problem, one of the research trends in the application of PANI is toward the formation of polyaniline composite materials in which PANI is embedded in an insulator polymer matrix such as polymethacrylate (Wan et al 1995; Cadenas and Hu 1998), polyvinyl carbazole (Cadenas and $\mathrm{Hu}$ 1998), polyvinyl alcohol (Chen and Fang 1991; Ogura et al 1997), nylon 6 (Byun and Im 1995, 1998), polycarbonate (Liu and Yang 1991) etc. The use of the insulator provides protection to PANI to improve its mechanical and chemical properties (Hu et al 1999).

In this paper, we have tried to show a direct relationship between the dielectric constant and processability of the synthesized PANI in a binary solvent medium of $N, N$-dimethyl formamide and water. The processable PANI synthesized at an optimum volume ratio of binary solvent mixture was deposited on an insulating host polymer PVA crosslinked with maleic anhydride and precoated on glass slide and was further used to investigate its electrical and gas sensor property.

\section{Experimental}

\subsection{Materials}

Reagent grade aniline hydrochloride (Merck, India) and ammonium persulphate $\left((\mathrm{NH})_{4} \mathrm{~S}_{2} \mathrm{O}_{8}\right)$ (Merck, India), polyvinyl alcohol (Fluka, Germany) (Mol wt), maleic anhydride (LOBA Chemie) were used without further purification. $N, N$-dimethyl formamide (Merck, India) was dried and used as solvent.

\subsection{Synthesis of polyaniline at different proportions of solvent dimethyl formamide and water}

Polyaniline was synthesized by the oxidation of aniline hydrochloride $(2.59 \mathrm{~g}, 20 \mathrm{mmol})$ and ammonium persulphate $(5.71 \mathrm{~g}, 25 \mathrm{mmol})$ with the oxidant and monomer ratio of 1.25:1. A binary solvent medium of $N, N$-dimethyl formamide (DMF) and water (total volume $100 \mathrm{ml}$ ) was chosen with the volume ratio of DMF and water varied as 1:0, 9:1, $8: 2,7: 3,6: 4,1: 1,4: 6,3: 7,2: 8,1: 9$ and $0: 1$. Each of the polymerization reaction was carried out at $-5^{\circ} \mathrm{C}$ and the reaction medium was stirred for $1 \mathrm{~h}$ and kept for $48 \mathrm{~h}$ for complete polymerization.

\subsection{Measurement of dielectric constants of different polymerization reaction medium}

A cylindrical dielectric cell was fabricated for measurement of small volume of each of the polymerization reaction medium $(4.8 \mathrm{ml})$. The cell contains aluminum electrodes with a surface radius of $1.25 \mathrm{~cm}$ and the distance between the electrodes is $1 \mathrm{~cm}$. Through aluminum, used as guard electrodes two copper leads were taken out to measure the capacitance by a HP Precision 4284A LCR meter $(20 \mathrm{~Hz}$ to $1 \mathrm{MHz}$ ). The electrical capacitance of the empty cell was about $30 \mathrm{pF}$. The static permittivities of the standard liquids were used to calibrate the dielectric cell. Measurement of capacitance of each of the reaction solution was performed at a frequency range of $1 \mathrm{~Hz}$ to $1 \mathrm{MHz}$. Electrical capacitance data were used to calculate the static permittivities of the solutions. The local spatial redistribution of polarized electrical charges in a material sample under an applied electric field is characterized by its dielectric constant (dielectric permittivity relative to the free space) $\varepsilon^{*}=\varepsilon-j \varepsilon_{\mathrm{r}}$, where $j^{2}=-1$. The real part $\varepsilon$ relates to the behaviour of an ideal insulator and characterizes the degree of electric polarizability of the material while the imaginary exponent, $\varepsilon_{\mathrm{r}}$, is associated with the electric energy dissipation into heat, due to electrical conduction and polarized charge fluctuation and is responsible for the phase difference between the applied alternating electric field and the polarization (Chong et al 2004). The constant value of the capacitance at high frequency indicates that the imaginary exponent, $\varepsilon_{\mathrm{r}}$, is zero at that frequency (Singh et al 1997) and medium permittivity can be calculated from the capacitance directly as $\varepsilon=$ $C(A / d)$, where $C$ is the capacitance, $A$ the cross sectional area and $d$ the distance between the electrodes. The dielectric constant measured by $\varepsilon_{d}=\varepsilon / \varepsilon_{0}$, where $\varepsilon_{0}$ is the permittivity of vacuum $\left(8.854 \times 10^{-12} \mathrm{farad} / \mathrm{m}\right)$.

\subsection{Film casting}

The green processable polyaniline solution was deposited for $1 \mathrm{~h}$ on a glass slide precoated with 5\% PVA crosslinked with maleic anhydride. The crosslinking of PVA with MA was done to reduce the water absorption characteristics of the host polymer PVA. The coating of PVA on the microscopic glass slides was done by running PVA solution over the glass and allowing it to dry for $6 \mathrm{~h}$ at $60^{\circ} \mathrm{C}$. The PANI was deposited on the PVA-coated glass slides by manual dipping in the PANI solution and extracting after $1 \mathrm{~h}$. For longer deposition time it was found that the film surface tends to crack while drying. The deposited polyaniline was dried in vacuum for $12 \mathrm{~h}$ at $70^{\circ} \mathrm{C}$. The dried sample was then washed several times with deionized water and finally dried in vacuum at $50^{\circ} \mathrm{C}$ for $6 \mathrm{~h}$. A uniform PANI film on glass slide was obtained for electrical study. This doped PANI film deposited on crosslinked PVA-coated glass slide would be depicted as 'deposited PANI film' in the following communication.

\subsection{Electrical property}

Four-probe set up, fabricated in our laboratory was used to measure the resistivity of the sample. According to 
four-point probe method the resistivity can be calculated using the relation, $\rho=2 \pi S\left(\frac{V}{I}\right)$, where, $S$ is the probe spacing $(\mathrm{mm})$, which was kept constant, $I$ the supplied current in $\mathrm{mA}$ and the corresponding voltage was measured in $\mathrm{mV}$. The conductivity can be calculated using the relationship, $\sigma=1 / \rho$.

\subsection{Gas sensor study}

In our gas sensor set up, the ammonia-air mixture was taken from the headspace of a bottle containing ammonia solution. The ammonia concentration in the mixture was estimated by trapping a known volume in ice-cold dilute hydrochloric solution that was titrated with standard sodium hydroxide before and after ammonia trapping (Prasad et al 2005). The polyaniline film specimen $(1 \times 1 \mathrm{~cm})$ was attached on a Bakelite sheet and then four contacts were made on the specimen by copper wires and silver paste with $2 \mathrm{~mm}$ distance between the probes. A constant current source (Keithley Model 224) and multimeter (Keithley model 196) were used to apply the current and to measure the voltage drop, respectively. A known volume of the ammonia gas was introduced (concentration calibrated to be at $100 \mathrm{ppm}$ ) and the change of resistance was monitored at every $30 \mathrm{~s}$ allowing the reading to stabilize. After some time when the response $\left(R / R_{0}\right)$ became saturated, the ammonia gas flow was stopped and air was passed to allow the sensor element to restore to the original state. This span covered one cycle of gas exposure. Here $R$ and $R_{0}$ were the resistances measured in the presence of ammonia and air, respectively. The sensitivity of the sensor was measured as the corresponding $R / R_{0}$ value when the response curve reached the plateau level. The response time was measured as the time in between the injection of the ammonia gas to the polyaniline sensor to the saturation of its response. The recovery time was the time in between the saturation response to the initial value in the presence of air (Prasad et al 2005).

\section{Results and discussion}

\subsection{Polyaniline synthesis at different solvent proportions of dimethyl formamide and water}

Polyaniline was synthesized in solvent medium at different volume proportions of $N, N$-dimethyl formamide and water. The objective was to study the processability of polyaniline with changing dielectric constant of the binary solvent medium. The dielectric constant as well as the dipole moment changes with different proportions of solvents mixtures and this has an effect on the molecular association of the polymer formed and hence processability (Ghanadzadeh et al 2005). In pure DMF no green colour characterizing the emeraldine salt was visible and the reaction medium was oily. With increasing water proportion the green colour emerges and only at a 1:1 volume

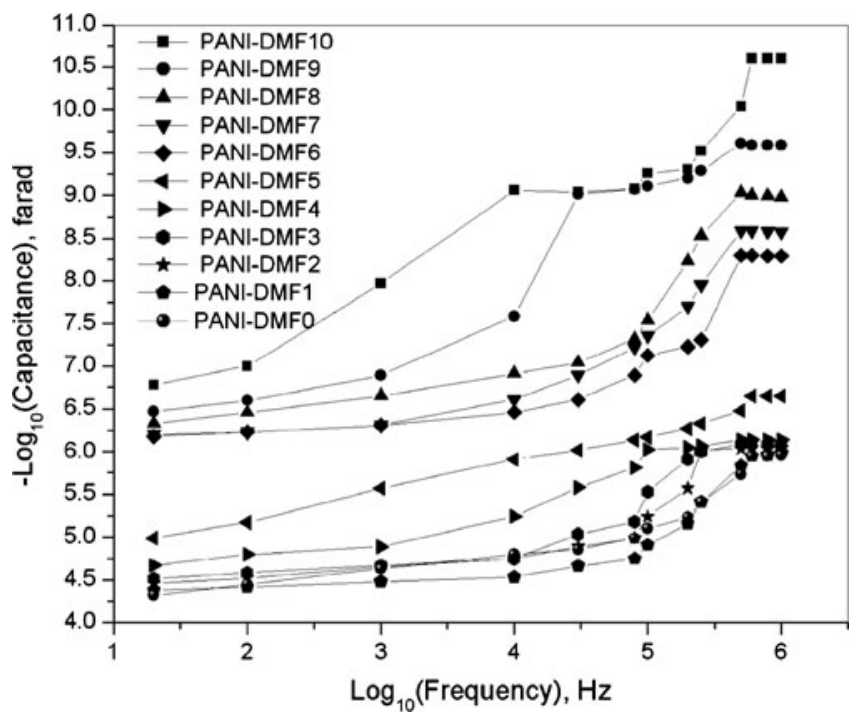

Figure 1. Capacitance change of different solvent binary mixtures of polymerization reaction medium with frequency (PANIDMF 10 denotes polymerization medium of pure DMF while PANI-DMF 0 denotes pure water as solvent).

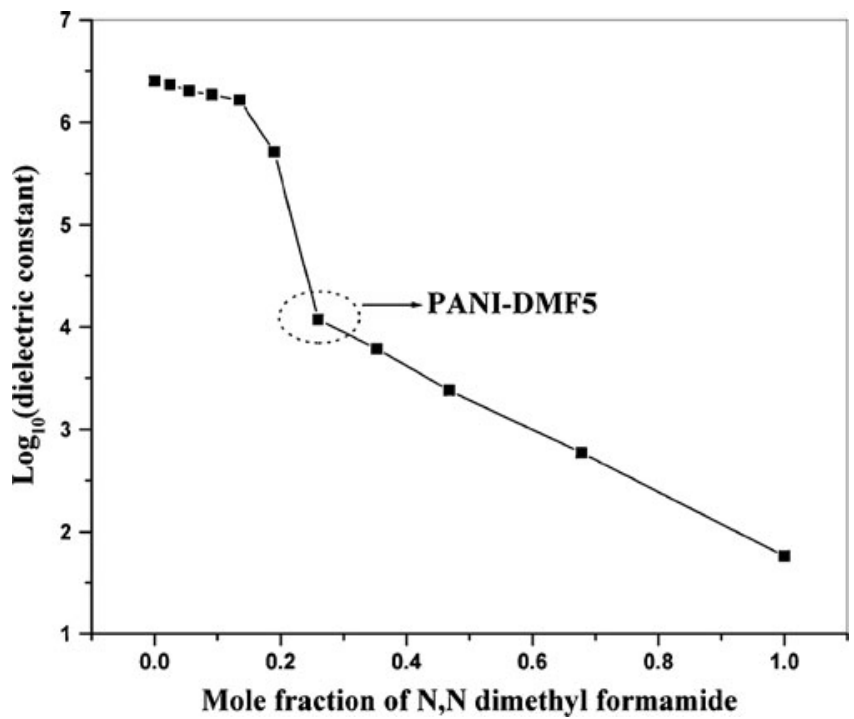

Figure 2. Change of dielectric constant of polymerization medium with change in mole fraction of DMF in solvent binary mixture. The permittivity of polymerization medium was calculated at a frequency of $1 \mathrm{MHz}$.

proportion of the solvent mixture a good suspension of the green PANI was visible. We call the solution as suspension as no film-forming characteristic of the processable solution was observed when the solvent was evaporated out. Only when the solid residue converted to base form and its solution in NMP was cast on a flat plate we got a uniform film. This observation shows that some polymer has been formed in the polymerization reaction. On further increase of water in the solvent mixture a tendency of precipitation of the polyemeraldine salt was observed. The reason for this behaviour 
Table 1. Dielectric constant and relative dielectric constant calculated for polyaniline polymerized at different volume proportions of binary solvent medium of DMF and water.

\begin{tabular}{|c|c|c|c|c|c|c|c|}
\hline Sample type & $\begin{array}{l}\text { Volume } \\
\text { of DMF } \\
(\mathrm{ml})\end{array}$ & $\begin{array}{l}\text { Volume } \\
\text { of water } \\
\quad(\mathrm{ml})\end{array}$ & $\begin{array}{c}\text { Mole } \\
\text { fraction } \\
\text { of DMF }\end{array}$ & $\begin{array}{c}\text { Capacitance } \\
\text { at } 1 \mathrm{MHz}\end{array}$ & $\begin{array}{l}\text { Medium } \\
\text { permittivity, } \varepsilon \\
(\mathrm{F} / \mathrm{m})\end{array}$ & $-\log _{10} \varepsilon$ & $\begin{array}{l}\text { Dielectric } \\
\text { constant, } \\
\varepsilon_{\mathrm{d}}=\varepsilon / \varepsilon_{0}\end{array}$ \\
\hline PANI-DMF10 & 100 & 0 & 1.000 & $024.86 \mathrm{pF}$ & $5.067 \times 10^{-10}$ & $9 \cdot 29$ & $5.722 \times 10^{1}$ \\
\hline PANI-DMF9 & 90 & 10 & 0.678 & $256.58 \mathrm{pF}$ & $5.230 \times 10^{-9}$ & $8 \cdot 28$ & $5.906 \times 10^{2}$ \\
\hline PANI-DMF8 & 80 & 20 & 0.468 & $001.04 \mathrm{nF}$ & $2.120 \times 10^{-8}$ & $7 \cdot 67$ & $2.394 \times 10^{3}$ \\
\hline PANI-DMF7 & 70 & 30 & $0 \cdot 353$ & $002.65 \mathrm{nF}$ & $5.401 \times 10^{-8}$ & $7 \cdot 26$ & $6 \cdot 100 \times 10^{3}$ \\
\hline PANI-DMF6 & 60 & 40 & $0 \cdot 260$ & $005 \cdot 13 \mathrm{nF}$ & $1.046 \times 10^{-7}$ & 6.98 & $1.181 \times 10^{4}$ \\
\hline PANI-DMF5 & 50 & 50 & $0 \cdot 190$ & $223.87 \mathrm{nF}$ & $4.563 \times 10^{-6}$ & $5 \cdot 34$ & $5 \cdot 154 \times 10^{5}$ \\
\hline PANI-DMF4 & 40 & 60 & $0 \cdot 135$ & $717.78 \mathrm{nF}$ & $1.463 \times 10^{-5}$ & 4.83 & $1.652 \times 10^{6}$ \\
\hline PANI-DMF3 & 30 & 70 & 0.091 & $806 \cdot 84 \mathrm{nF}$ & $1.644 \times 10^{-5}$ & 4.78 & $1.857 \times 10^{6}$ \\
\hline PANI-DMF2 & 20 & 80 & $0 \cdot 055$ & $880 \cdot 84 \mathrm{nF}$ & $1.795 \times 10^{-5}$ & 4.74 & $2.027 \times 10^{6}$ \\
\hline PANI-DMF1 & 90 & 90 & 0.025 & $001 \cdot 01 \mu \mathrm{F}$ & $2.059 \times 10^{-5}$ & 4.68 & $2.325 \times 10^{6}$ \\
\hline PANI-DMF0 & 0 & 100 & $0 \cdot 000$ & $001 \cdot 10 \mu \mathrm{F}$ & $2.532 \times 10^{-5}$ & $4 \cdot 65$ & $2.532 \times 10^{6}$ \\
\hline
\end{tabular}

Table 2. Dielectric constant, $\mathrm{pH}$ before and after polymerization reaction, yield of polyaniline synthesized in different volume proportions of water and DMF.

\begin{tabular}{lccccc}
\hline Sample type & $\begin{array}{c}\text { Dielectric constant, } \\
\varepsilon_{\mathrm{d}}=\varepsilon / \varepsilon_{0}\end{array}$ & $\mathrm{pH}_{0}$ & $\mathrm{pH}_{48}$ & $\mathrm{pH}=\left(\mathrm{pH}_{0}-\mathrm{pH}_{48}\right)$ & $\begin{array}{r}\text { Yield } \\
(\%)\end{array}$ \\
\hline PANI-DMF10 & $5.722 \times 10^{1}$ & 1.30 & 1.29 & 0.01 & $2 \cdot 5$ \\
PANI-DMF9 & $5.906 \times 10^{2}$ & 1.32 & 1.30 & 0.02 & $5 \cdot 1$ \\
PANI-DMF8 & $2.394 \times 10^{3}$ & 1.87 & 1.68 & $0 \cdot 19$ & 28.5 \\
PANI-DMF7 & $6.100 \times 10^{3}$ & 1.91 & 1.66 & 0.25 & $38 \cdot 7$ \\
PANI-DMF6 & $1.181 \times 10^{4}$ & 1.95 & 1.44 & 0.51 & $50 \cdot 8$ \\
PANI-DMF5 & $5.154 \times 10^{5}$ & 2.02 & 1.21 & 0.81 & $70 \cdot 7$ \\
PANI-DMF4 & $1.652 \times 10^{6}$ & 2.04 & 1.10 & 0.94 & $80 \cdot 2$ \\
PANI-DMF3 & $1.857 \times 10^{6}$ & 2.07 & 1.04 & 1.03 & $85 \cdot 3$ \\
PANI-DMF2 & $2.027 \times 10^{6}$ & 2.10 & 1.02 & 1.08 & 87.9 \\
PANI-DMF1 & $2.325 \times 10^{6}$ & $2 \cdot 10$ & 1.02 & 1.08 & 88.7 \\
PANI-DMF0 & $2.532 \times 10^{6}$ & 2.11 & 1.01 & 1.10 & 89.5 \\
\hline
\end{tabular}

might be, with increasing water content of the solvent mixture, dielectric constant of the medium increases facilitating higher polymer association and hence precipitation. Moreover, DMF has a polar - NH group conjugated with the adjacent $-\mathrm{C}=\mathrm{O}$ which being more compatible with the grown polymer intermingles with the adjacent polymer chain and restricts aggregation and phasing out.

\subsection{Measurement of dielectric constant}

The dielectric measurement has been shown to be a useful technique in characterizing the molecular ordering and the value of dielectric constant is strongly related both to the chemical structure of a molecule and to intermolecular interactions (Smyth 1980; Ghanadzadeh et al 2003; Ghanadzadeh and Beevers 2002, 2003). The measurement of capacitance with frequency of the different polymerization reaction medium by changing the volume concentration of DMF and water binary mixture is shown in figure 1 . The figure clearly shows that for all solutions the capacitance becomes constant at high frequency $(1 \mathrm{MHz})$, which offsets the electric energy dissipation by conduction. Figure 2 shows the change in the negative of logarithm of the dielectric constant or permittivity of the different polymerization medium with the change in the mole fraction of DMF (according to the change in volume ratios of DMF in different solutions). The dielectric constant increases with higher water content showing higher polymer chain association (Joo et al 1994). The polymerization medium with solvent proportion of 1:1 shows an intermediate dielectric constant showing a balance of molecular association and better processability. All the dielectric constants of the polymerization medium were measured at the frequency of $1 \mathrm{MHz}$ where the measured capacitance value becomes constant as shown in figure 1 . The constant value of the capacitance indicates that the imaginary component $\varepsilon_{\mathrm{r}}$ is zero at that frequency (Singh et al 1997) and the dielectric 
constant or the permittivity of the polymerization medium can be calculated from the relation, $\varepsilon=C(A / d)$, where $C$ is the capacitance, $A$ the cross-sectional area and $d$ the distance between the electrodes. Table 1 shows the dielectric constant and relative dielectric constant calculated for polyaniline polymerized at different volume proportions of binary solvent medium of $\mathrm{DMF}$ and water. The relative dielectric constant measured by $\varepsilon_{\mathrm{r}}=\varepsilon / \varepsilon_{0}$, where $\varepsilon_{0}$ is the dielectric constant of vacuum $\left(8.854 \times 10^{-12}\right)$. The relative dielectric constant of the polymerization medium changes from $5.722 \times 10^{1}$ in case of pure DMF to $2.532 \times 10^{6}$ in case of pure water as solvent (see table 2). The drastic increase of relative dielectric constant with increase in water content clearly shows more chain association and phasing out of the polyaniline. The capacitance of the polymerization medium (PANI-DMF5) measured at $1 \mathrm{MHz}$ frequency with a 1:1 volume proportion of DMF and water was found to be $223.87 \mathrm{nF}$ and the relative dielectric constant as $5 \cdot 154 \times 10^{5}$. Figure 2 shows that PANI-DMF5 has an intermediate value in the descending plot showing an optimum volume proportion of solvent for polyaniline growth and processability.

\subsection{UV-vis analysis of reaction solution at different solvent medium}

The UV-vis spectra of the polyaniline synthesized only in water, DMF and different volume ratios of DMF and water as binary solvent in the polymerization medium are shown in figure 3(a). UV-vis measurements of the different solutions were done in Mikropack UV-VIS-NIR, DH 2000. Each solution was diluted to same concentration to observe the change in intensity of the absorption peaks. The magnified picture of the $\pi-\pi^{*}$ transition and exciton peaks are shown in figures 3(b) and (c), respectively. The peak intensity and absorption wavelength values are shown in table 3 . Both $\pi-\pi^{*}$ transition and benzenoid to quinoid exciton transition peaks were observed as reported in standard literature (Wei et al 1994; Zheng et al 1997; Wu et al 2001). However, no $\pi-\pi^{*}$ transition peak was found in pure DMF solvent (PANI-DMF0) and DMF-water proportion of 9:1 (PANIDMF1) and also a progressive existence of $\pi-\pi^{*}$ transition peak was observed with increase in water content. The above finding shows that the low dielectric constant $(\varepsilon=38.3$ $\mathrm{F} / \mathrm{m}$ ) of the solvent DMF might hinder the oxidation and chain growth (Kulkarni et al 2004). However, while increasing the solvent dielectric constant by adding water $(\varepsilon=80$ $\mathrm{F} / \mathrm{m}$ ) an increase in the intensity of $\pi-\pi^{*}$ transition peak was observed which indicates extended growth and conjugation (Leclerc et al 1993). By increasing the proportion of water in the solvent mixture a red shift of the exciton peak was observed, which further indicates higher chain growth and assemblage (Leclerc et al 1993). Since higher chain assemblage results in ring torsion (Yang and Jing 2005) a blue shift of the $\pi-\pi^{*}$ transition band was observed in solvent mixtures of high water content. Overall, the UV-vis spectra gives a
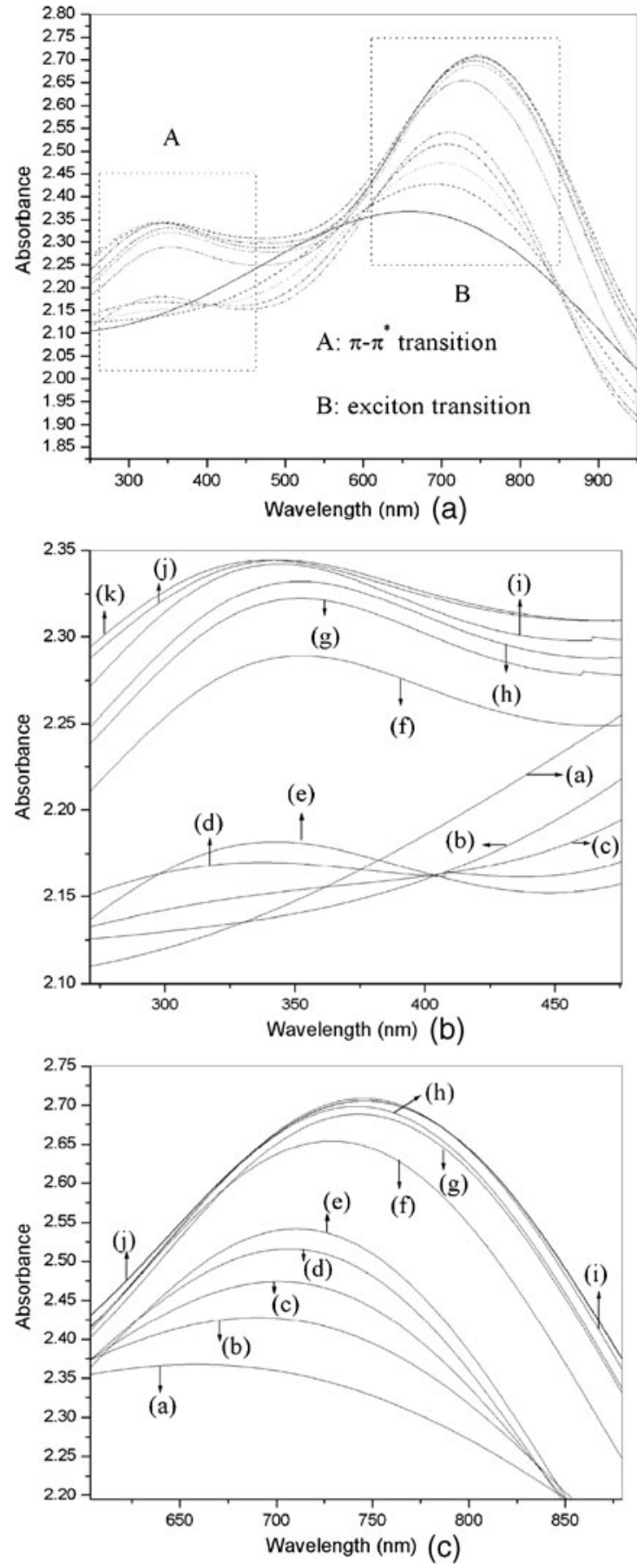

Figure 3. (a) UV spectra of synthesized polyaniline in different volume proportions of DMF and water as solvent medium, (b) magnified picture of $\pi-\pi^{*}$ transition of UV spectra depicting region A of (a): (a) PANI-DMF10, (b) PANI-DMF9, (c) PANI-DMF8, (d) PANI-DMF7, (e) PANI-DMF6, (f) PANI-DMF5, (g) PANIDMF4, (h) PANI-DMF3, (i) PANI-DMF2, (j) PANI-DMF1, (k) PANI-DMF0 and (c) magnified picture of exciton transition of UV spectra depicting region A of (a): (a) PANI-DMF10, (b) PANI-DMF9, (c) PANI-DMF8, (d) PANI-DMF7, (e) PANIDMF6, (f) PANI-DMF5, (g) PANI-DMF4, (h) PANI-DMF3, (i) PANI-DMF2, (j) PANI-DMF1, (k) PANI-DMF0. 
Table 3. UV spectral wavelength and peak height of maximum absorbance for different polymerization reaction medium.

\begin{tabular}{|c|c|c|c|c|}
\hline Sample type & $\begin{array}{l}\lambda \max \text { for } \pi-\pi^{*} \\
\text { transition }(\mathrm{nm})\end{array}$ & $\begin{array}{l}\text { Absorption } \\
\text { peak height }\end{array}$ & $\begin{array}{c}\lambda_{\max } \text { for exciton } \\
\text { transition }(\mathrm{nm})\end{array}$ & $\begin{array}{l}\text { Absorption } \\
\text { peak height }\end{array}$ \\
\hline PANI-DMF10 & - & - & $650 \cdot 65$ & $2 \cdot 37$ \\
\hline PANI-DMF9 & - & - & 687.89 & 2.42 \\
\hline PANI-DMF8 & $310 \cdot 49$ & $2 \cdot 15$ & 698.55 & $2 \cdot 47$ \\
\hline PANI-DMF7 & $315 \cdot 76$ & $2 \cdot 16$ & $706 \cdot 46$ & $2 \cdot 51$ \\
\hline PANI-DMF6 & 334.44 & $2 \cdot 18$ & $709 \cdot 20$ & $2 \cdot 55$ \\
\hline PANI-DMF5 & 344.99 & 2.29 & $727 \cdot 77$ & 2.65 \\
\hline PANI-DMF4 & $342 \cdot 35$ & $2 \cdot 32$ & 738.43 & $2 \cdot 68$ \\
\hline PANI-DMF3 & $339 \cdot 71$ & $2 \cdot 33$ & 741.07 & $2 \cdot 69$ \\
\hline PANI-DMF2 & 334.44 & $2 \cdot 34$ & 742.70 & 2.70 \\
\hline PANI-DMF1 & $331 \cdot 80$ & $2 \cdot 34$ & $743 \cdot 15$ & 2.70 \\
\hline PANI-DMF0 & $330 \cdot 05$ & $2 \cdot 34$ & 743.71 & 2.71 \\
\hline
\end{tabular}

plausible indication of the nature of intermolecular forces in the polyaniline chain, its growth and aggregation with the change in the dielectric of the binary solvent mixture.

\subsection{FTIR spectra of processable PANI film}

The PANI synthesized in 1:1 DMF and water solvent medium (PANI-DMF5) was deposited on PVA crosslinked with maleic anhydride coated glass slide and ATR spectrum was taken from Thermo Nicolet Nexus 870. The ATR spectrum as shown in figure 4 is nearly similar with the ATR spectrum of pristine PANI film which is PANI film doped by $1 \mathrm{M} \mathrm{HCl}$ according to our previous communication (Sengupta et al 2006). The hydrogen bonding with support of PVA matrix results in red shift of the

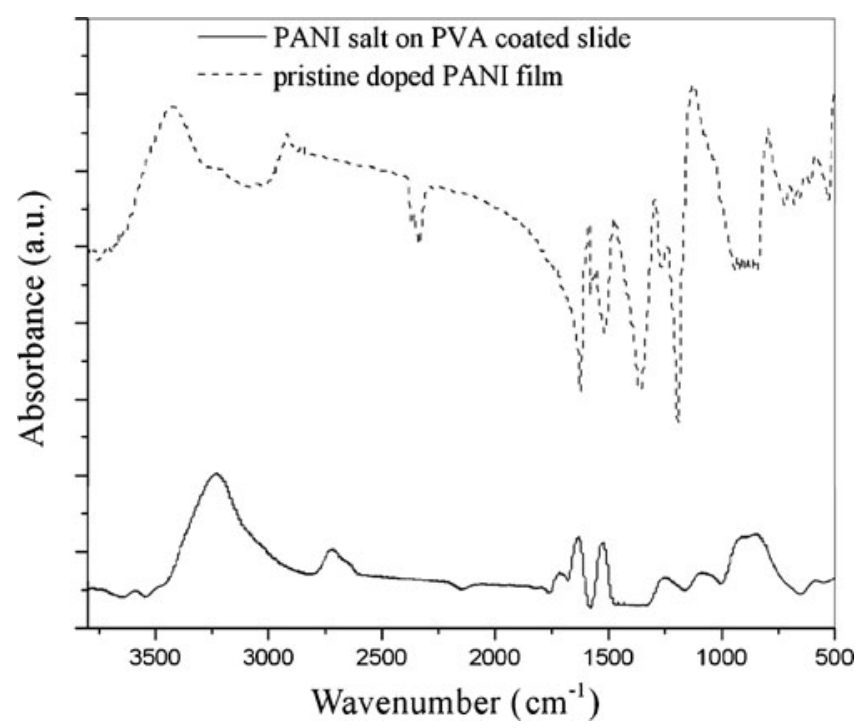

Figure 4. ATR spectra of pristine PANI film and PANI synthesized from DMF and water at volume ratio 1:1 and deposited on crosslinked PVA coated glass slide. absorption bands at $3300-2800 \mathrm{~cm}^{-1}$ reflecting the organization of the PANI chains within the film by hydrogen bonding involving $\mathrm{NH}$ and $\mathrm{NH}^{+}$groups (Malinauskas and Holze 1998; Yang and Jing 2005). The mutual PANI-PVA structural modifications also lead to significant shift of benzenoid and quinoid absorption bands (1560 and $1472 \mathrm{~cm}^{-1}$ ), respectively for pristine PANI to higher wavenumbers (1642 and $\left.1525 \mathrm{~cm}^{-1}\right)$, respectively for PANI on PVA matrix polymer (Ghosh et al 2001). The carbonyl stretching band observed at $1736 \mathrm{~cm}^{-1}$ for PVA (Molyneux 1983) shifts to lower frequency of $1706 \mathrm{~cm}^{-1}$ for the PANI deposited on PVA coated film. Apart from this, other bands like the $\mathrm{C}-\mathrm{N}$ stretching of secondary amine $\left(1273 \mathrm{~cm}^{-1}\right)$; vibration modes of $-\mathrm{NH}^{+}=$structure due to protonation $\left(1108 \mathrm{~cm}^{-1}\right)$ and $\mathrm{C}-\mathrm{H}$ stretching vibration band similar to the characteristic bands of PANI synthesized from aqueous acid were also observed. However, some red and blue shifts of the latter bands were observed due to the structural alignment of PANI on crosslinked PVA coated glass slide.

\subsection{XRD of processable PANI film}

The X-ray diffraction of the deposited PANI (PANI-DMF5) film was done in Philips Holland Type PW 1710 using $\mathrm{Cu}$ $\mathrm{K}_{\alpha}(\lambda=1.542 \AA)$ and is shown in figure 5. The diffraction peaks at $2 \theta$ of $10.5^{\circ}, 14^{\circ}, 21.3^{\circ}$ and $25.45^{\circ}$ are characteristic of the crystalline phase of the emeraldine salt, referred in literature (Pouget et al 1991). The crystalline peaks in the PANI deposited on PVA coated glass substrate clearly show the dominant effect of the conducting PANI than the host insulating PVA matrix. When only crosslinked PVA coated glass slides were scanned by XRD a broad scattering centred at $2 \theta$ value of $18 \cdot 5^{\circ}$ was obtained which characterizes its amorphous behaviour (Alexander 1969).

\subsection{AFM study of processable PANI film}

The AFM study was performed in NSOM AFM $1000^{\mathrm{TM}}$ to analyze the morphology of the deposited PANI film. The 
AFM measurements were carried out in air at ambient conditions $\left(25^{\circ} \mathrm{C}\right)$ using tapping mode probes. The tapping mode etched silicon probe with a spring constant of 1-5 N/m, nominal radius of curvature of $<10 \mathrm{~nm}$ and with resonance frequency of $270 \mathrm{kHz}$ was used. The $3 \mathrm{D}$ and $2 \mathrm{D}$ surface

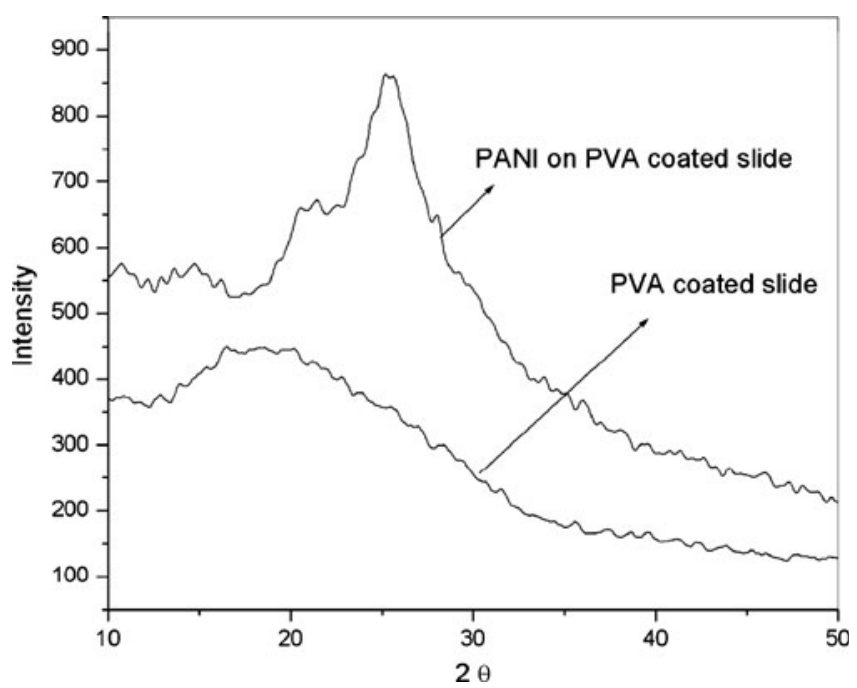

Figure 5. X-ray diffraction pattern of deposited PANI film and crosslinked PVA film coated on glass slide. topographies of the height images of deposited PANI (PANIDMF5) on crosslinked PVA coated glass surface are shown in figures 6(a) and (b). The film deposited is found to be relatively flat and homogenous having $14.5 \mathrm{~nm}$ average roughness of coated surface. The cluster size distribution is very

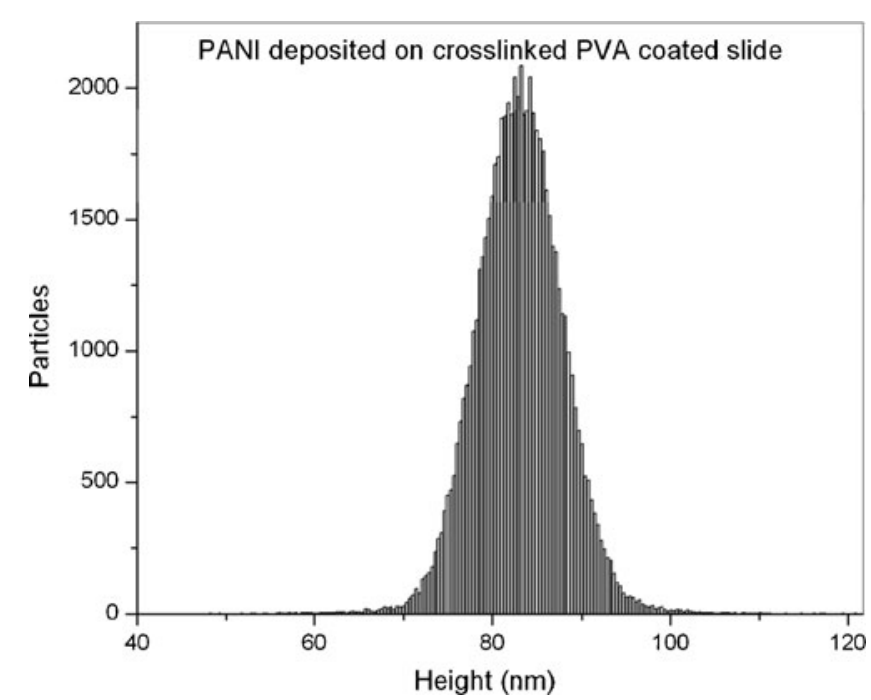

Figure 7. Cluster size distribution in deposited PANI film.
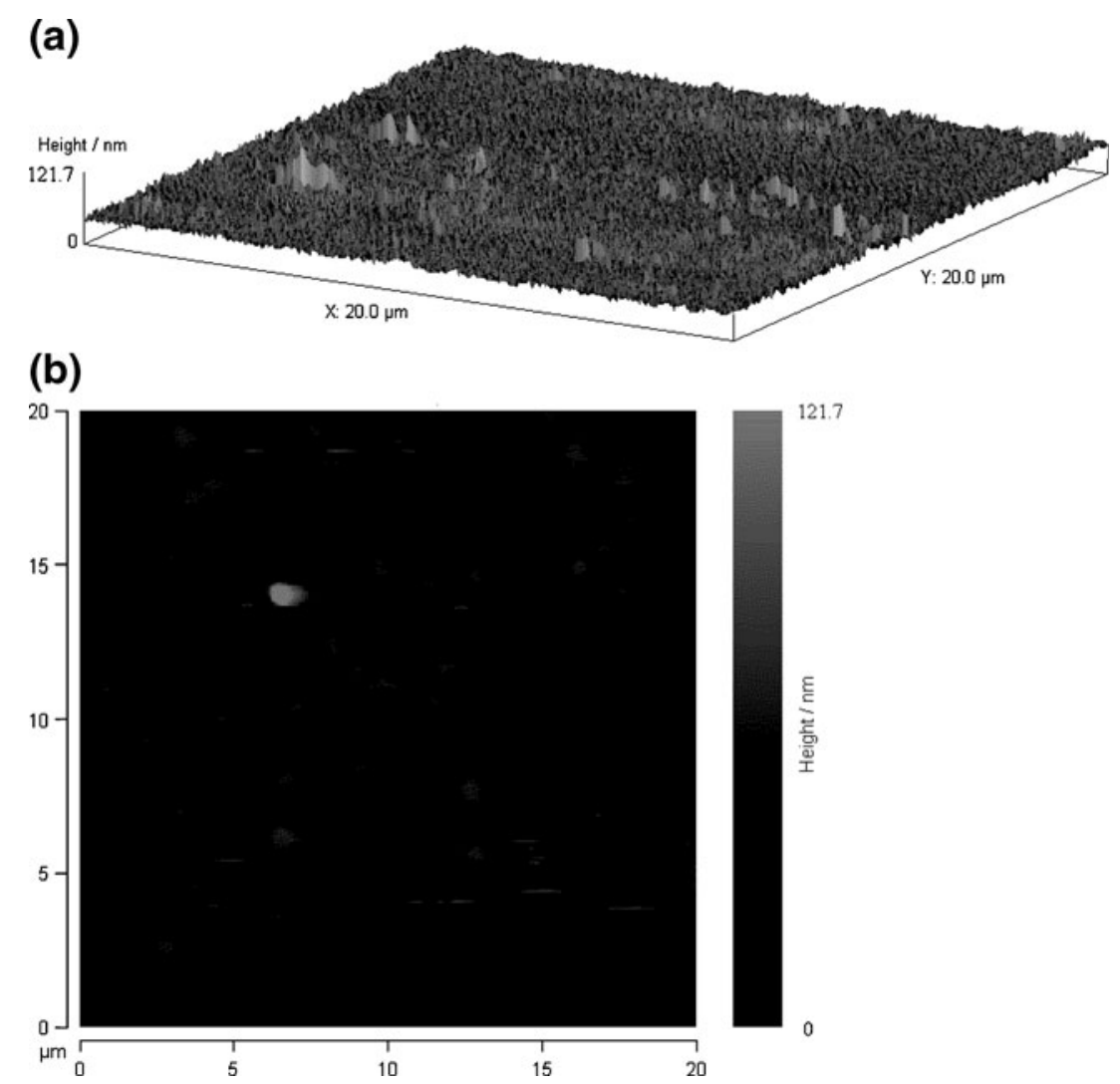

Figure 6. (a) 3-D AFM morphology of deposited PANI film and (b) 2-D AFM morphology of deposited PANI film. 


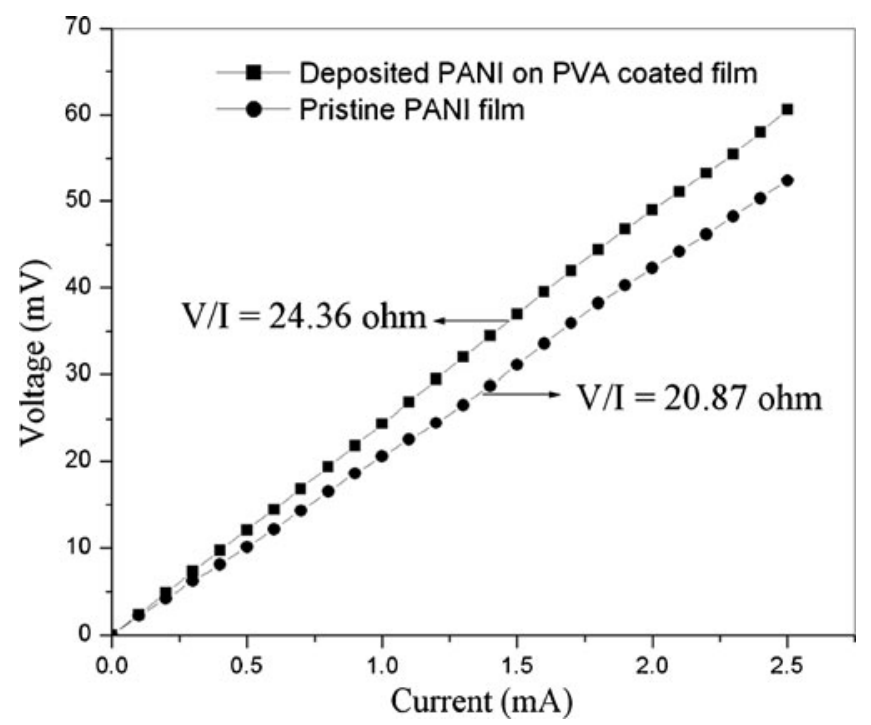

Figure 8. $\quad V-I$ characteristics of pristine PANI film and deposited PANI film (PANI-DMF5).

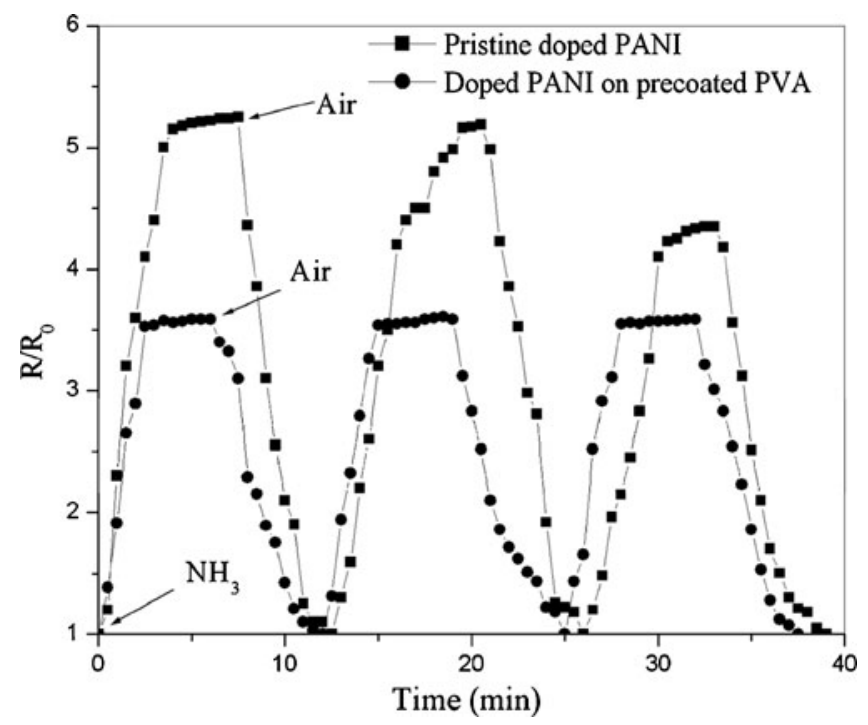

Figure 9. Ammonia sensing study of deposited (PANI-DMF5) and pristine PANI film for 3 cycles with an ammonia concentration of $100 \mathrm{ppm}$.

aptly depicted in the histogram of figure 7. A sharp cluster size distribution centred at around $85 \mathrm{~nm}$ and with a maximum cluster size of $105 \mathrm{~nm}$ is observed.

\subsection{D.C. conductivity measurement}

The $V / I$ characteristics of deposited PANI (PANI-DMF5) film and pristine PANI film are shown in figure 8 . The figure shows that the surface resistance of the deposited film is fairly same to $\mathrm{HCl}$ doped pristine PANI film (Sengupta et al 2006; Sengupta and Adhikari 2007). A little higher value of resistance of PANI-DMF5 is observed due to some surface

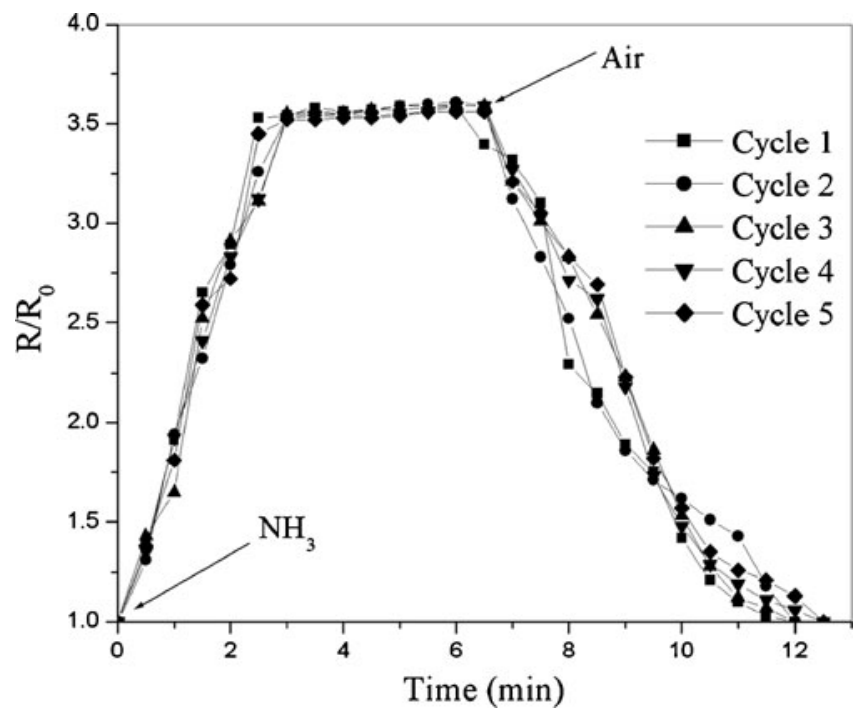

Figure 10. Sensing stability study of deposited PANI film (PANI-DMF5) for five repeated cycles with an ammonia concentration of $100 \mathrm{ppm}$.

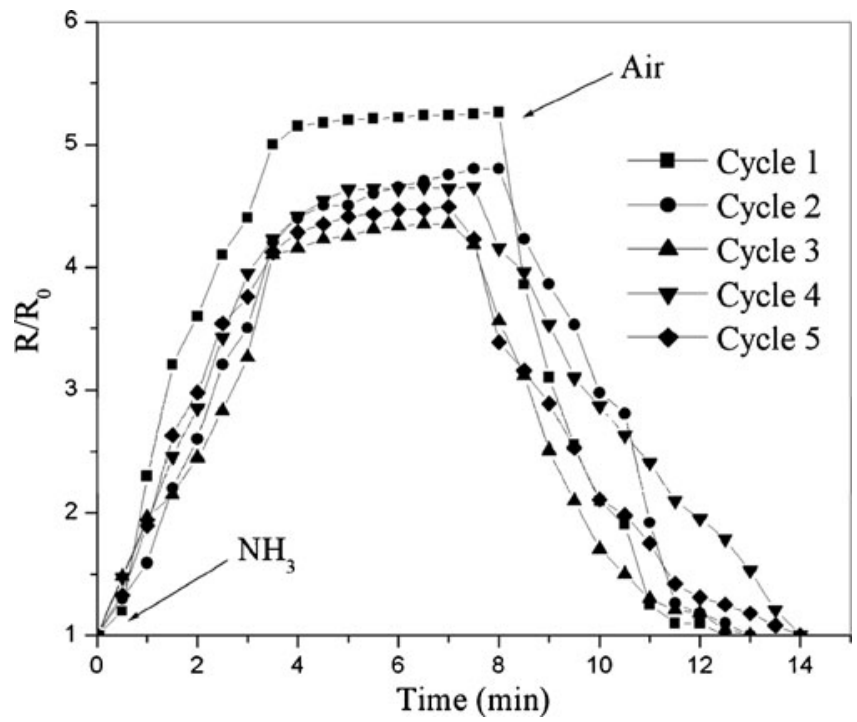

Figure 11. Sensing stability study of pristine PANI film for five repeated cycles with an ammonia concentration of $100 \mathrm{ppm}$.

inhomogeneity resulting from an insulating polymer matrix, which is also reflected in the 3-D surface (figure 6). The work function of the copper contacts being higher than that of the deposited film (Huang J et al 2003; Huang L M et al 2003) a fairly linear and ohmic contact is observed.

\subsection{Gas sensor study}

The ammonia gas sensing behaviour of the deposited PANI (PANI-DMF5) film as well as doped pristine PANI film is shown in figure 9. For the sensor, the increase in the 
Table 4. Comparison of change of sensitivity of PANI deposited film and pristine PANI with 5 different cycles.

\begin{tabular}{lcc}
\hline $\begin{array}{l}\text { Number of ammonia } \\
\text { gas cycle at a fixed } \\
\text { concentration of }\end{array}$ & $\begin{array}{c}\text { Sensitivity } \\
\text { of pristine } \\
\text { PANI film } \\
\left(R / R_{0}\right)\end{array}$ & $\begin{array}{c}\text { Sensitivity of PANI } \\
\text { deposited on PVA } \\
\text { coated glass slide } \\
\left(R / R_{0}\right)\end{array}$ \\
\hline 1 & 5.00 & 3.54 \\
2 & 4.40 & 3.54 \\
3 & $4 \cdot 10$ & 3.55 \\
4 & 4.28 & 3.53 \\
5 & 4.54 & 3.52 \\
\hline
\end{tabular}

resistance is observed after inflow of $\mathrm{NH}_{3}$ gas. This change in resistance can be attributed as the ammonia gas is chemisorbed; ammonium ions formed by the reaction with the proton of the emeraldine salt leads to localization of polarons on the conducting PANI and hence increase in resistance (Prasad et al 2005). On replacement of ammonia gas by air the ammonium ions decompose into ammonia leaving the proton back on the PANI chain. This is the desorption phenomenon of the sensor. The sensing mechanism is thus governed by the protonation-deprotonation phenomenon (Kukla et al 1996; Dhawan et al 1997; Koul et al 2001). In our study the response times, measured for the deposited PANI film and pristine PANI, are $3.5 \mathrm{~min}$ and $5 \mathrm{~min}$, respectively. The recovery times of the former two are $5.5 \mathrm{~min}$ and 6.5 min, respectively. This shows that the thin layer of PANI deposited on PVA host matrix gives a faster response and recovery than pristine PANI when exposed to ammonia gas. The reason may be as there is more accessibility for swelling of the pristine PANI, the volume change of the free standing film leads to some incoherent absorption and desorption process of chemisorbed ammonia (Lee et al 2003). Moreover, since the deposited film has an insulating host polymer the penetration of ammonia absorption is less than pristine PANI leading to faster recovery of ammonia gas from the sensor matrix.

The stabilities of the deposited PANI and pristine PANI films studied for five cycles are shown in figures 10 and 11 , respectively. It is found that the deposited PANI film shows a higher stability than the pristine PANI film. The change of sensitivity of both sensors with the number of cycles in table 4 clearly shows that the deposited PANI film on precoated crosslinked PVA has a better ammonia sensing performance.

\section{Conclusions}

In this work we have reported the synthesis of processable doped PANI by changing the dielectric constant of the binary solvent mixture of the polymerization medium. The solvent DMF had the added benefit of an amide functional group having affinity to the backbone of PANI, increasing the solute and solvent interaction and facilitating dissolution. The optimum volume ratio of DMF and water was found to be 1:1 in our polymerization condition to obtain processable doped PANI. Our next objective was to deposit the soluble PANI on a host polymer PVA to get a thin uniform film used as sensitive layer for ammonia sensing. PVA was crosslinked with maleic anhydride to reduce its hydrophilic property. The crosslinked PVA showed good compatibility with the coated PANI as evident from the morphology study of coated film by AFM. The deposited PANI film showed better sensor response in terms of repeatability and stability than that of pristine PANI film. The supporting insulating polymer matrix helped in better sensing response to conducting polymer sensitive layer as compared to the pristine film only.

\section{Acknowledgement}

We gratefully acknowledge the University Grants Commission, Government of India, for providing fellowships to the two authors (PPS and PK) during the tenure of the work.

\section{References}

Alexander L E 1969 X-ray diffraction methods in polymer science (Wiley Interscience) p. 43

Byun S W and Im S S 1995 J. Appl. Polym. Sci. 56425

Byun S W and Im S S 1998 Polymer 39485

Cadenas J and Hu H 1998 Solar Energy Mater. Solar Cells 55105

Chabukswar V V, Pethkar S and Athawale A A 2001 Sens. Actuators B77 657

Chen S -A and Fang W -G 1991 Macromolecules 241242

Chen S -A and Lee H -T 1995 Macromolecules 282858

Chong T F, Fabbri A, Guilbaud J P and Coussy O 2004 C.R. Mecanique 332639

Dannhauser W and Flucckinger A F 1964 J. Phys. Chem. 681814

Deng A, Cheng J and Huang H 2002 Anal. Chim. Acta 46149

Dhawan S K, Kumar D, Ram M K, Chandra S and Trivedi D C 1997 Sens. Actuators B40 99

Ghanadzadeh A and Beevers M S 2002 J. Mol. Liq. 10047

Ghanadzadeh A and Beevers M S 2003 J. Mol. Liq. 102365

Ghanadzadeh A, Mamaghani M and Anbir L 2003 J. Sol. Chem. 32 365

Ghanadzadeh A, Ghanadzadeh H, Sariri R and Ebrahimi L 2005 J. Chem. Thermodynamics 37357

Ghosh P, Siddhanta S K, Haque S R and Chakrabarti A 2001 Synth. Met. 12383

Hu H, Saniger J M and Bannelos J G 1999 Thin Solid Films 347241

Huang J, Virji S, Weiller B H and Kaner R B 2003 J. Am. Chem. Soc. 125314

Huang L M, Wen T C, Gopalan A and Ren R 2003 Mater. Sci. Eng. B104 88

Janini G M and Katrib A H 1983 J. Chem. Educ. 601087

Joo J, Oblakowski Z, Du G, Pouget J P, Oh E J, Wiesinger J M, Min Y and Epstein A G 1994 Phys. Rev. B49 2977 
Jousseaume V, Morsli M, Bonnet A, Tesson O and Lefrant S 1998a J. Appl. Polym. Sci. 671205

Jousseaume V, Morsli M, Bonnet A and Lefrant S 1998b J. Appl. Polym. Sci. 67209

Koul S, Chandra R and Dhawan S K 2001 Sens. Actuators B75 151

Kukla A L, Shirshov Y M and Piletsky S A 1996 Sens. Actuators B37 135

Kulkarni M V, Viswanath A K, Marimuthu R and Seth T 2004 J. Polym. Sci. Part A: Polym. Chem. 422043

Leclerc M, Aparno G D and Zotti G 1993 Synth. Met. 55-57 1527

Lee Y S, Joo B S, Choi N J, Liou J O, Huh J S and Lee D D 2003 Sens. Actuators B93 148

Lindsey S E and Street G B 1984 Synth. Met. 1067

Liu J -M and Yang S C 1991 J. Chem. Soc. Chem. Commun. 211529

Malinauskas A and Holze R 1998 Synth. Met. 9731

Molyneux P 1983 Water soluble polymers: Properties and behaviour (New York: CRC Press) Vol. 1, p. 128

Nylander C, Armgarth M and Lundstrom L 1983 in Proceedings of the international meeting on chemical sensors (eds) T Seiyama et al (Amsterdam: Elsevier) p. 203

Ogura K, Saino T, Nakayama M and Shiigi H 1997 J. Mater. Chem. 72363

Ojio T and Miyata S 1986 Polym. J. 1895

Planes J, Wolter A, Cheguettine Y, Pron A, Genoud F and Nechtschein M 1998 Phys. Rev. B58 7774
Pouget J P, Josefowicz M E, Epstein A J, Tang X and MacDiarmid A G 1991 Macromolecules 24779

Prasad G K, Radhakrishnan T P, Kumar D S and Krishna M G 2005 Sens. Actuators B106 626

Sengupta P P, Barik S and Adhikari B 2006 Mater. Manuf. Processes 21263

Sengupta P P and Adhikari B 2007 Mater. Sci. Eng. A459 278

Singh R, Arora V, Tandon R P, Mansingh A and Chandra S 1999 Synth. Met. 104137

Singh R, Narula A K, Tandon R P, Mansingh A and Chandra S 1997 Philos. Mag. B75 419

Smith J W 1955 Electric dipole moment (London: Butterworths) Ch. 5

Smyth C P 1980 Molecular interactions (New York: Wiley) Vol. II Sun L, Yang S C and Liu J -M 1994 Mater. Res. Soc. Symp. Proc. 328167

Tobolkova E, Prokes J, Krivka I, Trchova M and Stejskal J 2004 Macromol. Symp. 212447

Wan M, Li M, Li J and Liu Z 1995 Thin Solid Films 259188

Wei Y, Hsueh K F and Jang G W 1994 Macromolecules 27518

Wu C G, Yeh Y R, Chen J Y and Chiou Y H 2001 Polymer 42 2877

Yang Y and Jing X 2005 Polym. Test. 24153

Zheng W, Angelopoulos M, Epstein A J and Macdiarmid A G 1997 Macromolecules $\mathbf{3 0} 2953$ 